\title{
Polycystic ovarian syndrome in identical twins
}

\author{
C. HUTTON* \\ M.R.C.P.
}

\author{
F. CLARK \\ F.R.C.P.
}

Freeman Hospital, Newcastle-upon-Tyne NE7 7DN

\begin{abstract}
Summary
Two 21-year-old female twins are described. They presented simultaneously with hirsutism and oligomenorrhoea. Investigation showed they had polycystic ovaries, they were identical and were not cases of post-pubertal adrenogenital syndrome.
\end{abstract}

KEY WORDS: polycystic ovarian syndrome, twins, hirsutism, oligomenorrhoea.

\section{Case reports}

Case 1

She presented with a 10-month history of hirsutism, developing upper lip hair, sideboards, extension of the pubic escutcheon and oligomenorrhoea, with cycles varying between 6 and 7 weeks.

She had had a normal menarche aged 16 years, no previous illnesses and there was no family history of infertility, hirsutism or obesity. Two brothers had insulin-dependent diabetes. Her weight and secondary sexual characteristics were normal. There was no virilisation and she was normotensive. Pelvic examination showed bilateral enlargement of her ovaries. Ultrasound examination confirmed bilaterally enlarged cystic ovaries.

Her endocrine profile showed a mild elevation of plasma testosterone, androstenedione and cannulated prolactin. Urinary 17-oxo and 11-hydroxysteroids were also just higher than normal.

Plasma FSH, LH, 17- $\beta$-oestradiol, progesterone, 17- $\alpha$-hydroxyprogesterone, diurnal ACTH and cortisol were normal. The short synacthen test cortisol response was normal and a mild rise $(2.5$ to 7.2 $\mathrm{nmol} /$ litre) in 17- $\alpha$-hydroxyprogesterone occurred.

\section{Case 2}

She presented with 6-months hirsutism, with a generalized increase in body hair and 1-years oligomenorrhoea, cycles varying from 2 to 5 months. He menache at 16 years was normal. Secondary sexual

\footnotetext{
* Present address: Department of Medicine, Bristol Royal Infirmary, Bristol BS2 $8 \mathrm{HW}$.
}

development and external genitalia were normal $\vec{\circ}$ Pelvic examination revealed bilateral ovarian en $\vec{\jmath}$ largement. Ultrasound confirmed polycystic ovaries

She had a persistently high LH level, mildlo elevated testosterone, androstenedione and prolactin? 17-oxogenic and 11-hydroxysteroids were mildls elevated but 17-hydroxysteroids were normalo Plasma FSH, diurnal cortisol and the response to short synacthen test of cortisol and 17- $\alpha$-hydroxypro $\$$ gesterone were normal. The 17- $\alpha$-hydroxyprogestero one rose from $2 \cdot 1 \mathrm{nmol} /$ litre to $5 \cdot 5 \mathrm{nmol} /$ litre over $60^{\circ}$ $\min$.

Genetic testing at 20 loci showed identity to give probability of zygosity of 0.99957 . Karyotyping wit pit band Giemsa staining techniques showed a normat $46 \mathrm{XX}$ pattern and no evidence of mosaicism.

\section{Discussion}

Polycystic ovarian syndrome is an ill-defineo syndrome. Its features of oligomenorrhoea, infertil ity, obesity and hirsutism may occur without any cystic change in the ovary. Conversely polycystic ovaries occur without the associated features. The presence of mild endocrine abnormalities, in particular androgen excess, suggests that there may be की common pattern to the disease, but its diagnosis remains one of exclusion. There are no specific features or tests.

Virilising ovarian and adrenal tumours, Cushing syndrome and the adrenogenital syndrome must be excluded. Androgen levels are usually high in hormone secreting tumours. Cushing's syndrome loseso the normal cortisol diurnal variation. More proble matic is the exclusion of the adrenogenital syndrome.

There are three known blocks in adrenal cortisol production producing excessive androgen production due to the failure of cortisol to inhibit the ACTHN drive. Their presence should be demonstrated by a failure of cortisol to rise in response to ACTH, aftero the endogenous ACTH has been suppressed witho dexamethasone. 3- $\beta$-hydroxysteroid dehydrogenase block results in a failure to convert pregnenolone to $\stackrel{\infty}{+}$ progesterone increasing pregnanetriol excretion. 11- 0 $\beta$-hydroxylase deficiency causes a failure to converto 
deoxycortisone to corticosterone which results in the accumulation of deoxycortisone producing hypertension. 21-hydroxylase deficiency is the commonest type of the deficiency. It prevents hydroxylation of progesterone and 17- $\alpha$-hydroxyprogesterone to 11-deoxycortisol causing a rise in basal 17- $\alpha$ hydroxyprogesterone. Neither patient shows evidence of the homozygous state of these enzyme deficiencies.

The problem is, does the heterozygous carrier have identifiable abnormalities in cortisol synthesis that can cause hirsutism and could this present postpubertally? Adult manifestations of incomplete 21hydroxylase deficiency mimicking polycystic ovarian syndrome has been described by Lobo and Goebelsmann (1980). They identified a subgroup of patients with polycystic ovarian syndrome who show an excessive rise of 17- $\alpha$-hydroxyprogesterone to ACTH stimulation. A rise from $1 \mathrm{ng} / \mathrm{ml}$ to $90 \mathrm{ng} / \mathrm{ml}$ after 60 min. A rise was not documented in normal women in the luteal or follicular phase, or in other polycystic women. They also showed a decreased cortisol response but a normal 11-deoxycortisol response to ACTH. This pattern was not seen in the twins described.

The aetiology of the syndrome is obscure. There is some evidence it is congenital and it is in this context that its presence here in identical twins is particularly interesting. A familial tendency is described by Cooper et al. (1968) but this has not been noted in all series (Stein, 1964). There are a few reports of sisters developing it and two of identical twins being affected (Jeffcoate, 1964; Goldzieher and Green, 1962). Neither pair are described with details of the assessment of their diagnosis, endocrine profile nor genetic identity. We know of no reports in nonidentical twins.

Several workers have noted chromosomal abnormalities. Parker et al. (1980) describe a high incidence of mosaicism, particularly of the $\mathrm{X}$ chromosome and trisomy 14. Both are rare occurrences in the general population. Again not all authorities confirm this.
There is considerable interest in the role of androgens. Testosterone and androstenedione levels are usually high normal or mildly elevated and this may explain the virilisation. Moreover there is experimental animal evidence to show that polycystic ovaries result secondarily to increased circulating androgens. Adrenal, ovarian hypersecretion and increased dermal conversion have been postulated. However, the frequent occurrence of high LH levels, mild elevation of prolactin and exaggerated $\mathrm{LH}$ response to LRH suggest a more widespread incoordination of hormonal homeostatic control possibly secondarily to hypothalamic dysfunction. However, just as the clinical picture varies, the endocrine patterns are diverse and we do not as yet know how to match the two patterns.

It seems probable that polycystic ovarian syndrome will be gradually whittled down as the component diseases are identified and diagnostic criteria evolved. At present, we are in the dark. These twins demonstrate that the syndrome can occur in identical twins. How it does so remains unexplained.

\section{References}

CoOPER, H.E., SPellacy, W.N., PREM, R.A. \& Cohen, W.D. (1968) Hereditary factors in the polycystic ovarian syndrome. American Journal of Obstetrics and Gynecology, 100, 371.

COPE, C.L. (1972) Congenital adrenal hyperplasia. In: Adrenal Steroids and Disease, p. 355. Pitman Medical, London.

GolDZIEHER, J.W. \& GReEN, J.A. (1962) The polycystic ovary. Part I Clinical and histological features. Journal of Clinical Endocrinology, 22, 325.

JEFFCOATE, T.N. (1964) The androgenised ovary with special reference to the Stein-Leventhal syndrome. American Journal of Obstetrics and Gynecology, 88, 143.

PARKer, R., Ming, P., Rajan, R., Goodner, D. \& Reme, G. (1980) The clinical and cytogenic studies of patients with polycystic ovarian syndrome. American Journal of Obstetrics and Gynecology, 88, 143.

Lobo, R.A. \& GoebelsmanN, U. (1980) Adult manifestations of congenital adrenal hyperplasia mimicking the polycystic ovarian syndrome. American Journal of Obstetrics and Gynecology, 138, 720.

STEIN, I.F. (1964) Multiple pregnancy following wedge resection of the Stein-Leventhal Syndrome. International Journal of Fertility, 9, 343. 